\section{PTU-052 LONG TERM OUTCOME OF PATIENTS WITH REFLUX SYMPTOMS AND SYMPTOMATIC ESOPHAGEAL DYSFUNCTION DURING AND AFTER A STANDARDIZED TEST MEAL:A HIGH-RESOLUTION MANOMETRY STUDY}

doi:10.1136/gutjnl-2013-304907.144

1."R Sweis, ${ }^{2} \mathrm{G}$ Brady, ${ }^{3} \mathrm{~A}$ Anggiansah, ${ }^{1} \mathrm{~A}$ Lee, ${ }^{1} \mathrm{~A}$ Valdes, ${ }^{4} \mathrm{~T}$ Wong, ${ }^{5} \mathrm{M}$ Fox. ${ }^{1}$ Oesophageal lab; '2St Thomas' Hospital, London, UK; ${ }^{3}$ Oesophagael lab; ${ }^{4}$ Gastroenterology Department, St Thomas' Hospital, London; ${ }^{5} \mathrm{NIHR}$ Biomedical Research Unit and Digestive Diseases Centre, Nottingham University Hospitals, Nottingham, UK

Introduction Recently we presented novel methodology for the assessment of oesophageal function and symptoms during and after a standard test meal. ${ }^{1}$ In the absence of a "gold standard", outcome data provides insight into the clinical impact of this test in patients with reflux symptoms

Methods 18 patients referred for investigation of reflux symptoms and 10 healthy volunteers underwent High Resolution Manometry (HRM) with $5 \mathrm{ml}$ water, $200 \mathrm{ml}$ water drink and test meal followed by $10 \mathrm{~min}$ post-prandial observation. $24 \mathrm{hr} \mathrm{pH}$ studies were performed in patients. The number of Symptoms Associated with oesophageal Dysfunction( $\mathrm{SAD}$ ) was calculated. HRM findings and initial diagnosis were compared with the final diagnosis and outcome at 2 years

Results No symptoms occurred with $5 \mathrm{ml}$ water. 12/18(67\%) patients had SAD (mean SAD 2(range 0-7)) during/after the meal. Compared to $5 \mathrm{ml}$ water, manometric diagnosis was altered in $12 / 18(67 \%)$. No healthy volunteers had $\mathrm{SAD}$.

11/18 patients had GORD on $\mathrm{pH}$ studies. By 2 years, $5 / 11$ had anti-reflux surgery with excellent outcome. All 5 exhibited dysmotility (e.g. hypotensive/failed peristalsis) during the meal with symptomatic postprandial reflux events (transient lower oesophageal sphincter relaxation + common cavity). Of the $6 / 11$ with GORD who did not have surgery, 2 declined it despite pathological $\mathrm{pH}$ results and symptomatic reflux events after the meal; both remain symptomatic despite acid-reducing therapy. The remaining 4 of 6 patients also had symptomatic dysmotility but were not offered surgery. 2 with severe hypotensive dysmotility and symptomatic reflux responded to acid suppression. 1 with (peptic) outlet obstruction and 1 with diffuse spasm did not respond to medication.

Of the 7 with functional heartburn (negative $\mathrm{pH}$ results), 2 who had normal HRM responded to dietary/stress management. 2 with symptomatic reflux during HRM had good response to acid-suppression (i.e. false neg $\mathrm{pH}$ study). The final 3 with reflux-like symptoms had outflow obstruction identified only during the meal; 1 had good outcome after dilatation, 1 was too frail for therapy and remains symptomatic and the last was lost to follow-up.

Conclusion HRM studies which include a test meal and postprandial observation provide an objective explanation for symptoms in the majority of patients referred for investigation of "reflux" symptoms. Long-term follow-up suggests this information can guide management especially in patientswithout definitive diagnosis following standard $5 \mathrm{ml}$ water HRM and negative $\mathrm{pH}$-studies

Disclosure of Interest None Declared

\section{REFERENCE}

1. Sweis R et al. Gastro 2011; 140

\section{Inflammatory bowel disease}

\section{PTU-053 USEFULNESS OF FECAL CALPROTECTIN IN CLINICAL PRACTICE IN A DISTRICT GENERAL HOSPITAL}

doi:10.1136/gutjnl-2013-304907.145

1."A Malik, ${ }^{2} \mathrm{D}$ Bowen, ${ }^{3}$ I Rees. 'Gastroenterology, Prine Philip Hospital, Llanelli; ${ }^{2}$ Gastroenterology, West UK Hospital, Carmarthen; ${ }^{3}$ Gastroenterology, Prince Philip Hospital, Llanelli, UK
Introduction Calprotectin is a calcium and zinc binding protein mainly contained in neutrophils. If present in stools it is a marker of bowel inflammation. We evaluated the diagnostic value of faecal calprotectin (FC) as a non-invasive marker of bowel inflammation in routine out-patient gastroenterology clinic.

Methods A retrospective study was conducted of patients who had faecal calprotectin evaluated for various indications in outpatient gastroenterology clinic over a 12 month period. Presenting symptoms, FC results and the endoscopic findings were recorded. FC level more than $50 \mu \mathrm{g} / \mathrm{gm}$ was considered positive.

Results FC was requested for 72 patients. 44 were female (mean age 44 years) and 28 were male (mean age 47 years). FC was requested for various symptoms including chronic diarrhoea, abdominal pain, abdominal distension and per rectal bleeding. Patients were divided into 3 groups based on clinical practise of gastroenterologist.

In the first group FC alone was requested initially as a screening test to assess bowel inflammation. 31 patients fell in this group, 21 of 31 had negative FC and no further investigations were done, while 10 of 31 had positive FC (mean $150.3 \mu \mathrm{g} / \mathrm{gm}$ ). Out of these 5 had no further investigations as symptoms settled on subsequent clinic visit and 5 went on to have further investigations (Colonoscopy +/- Capsule endoscopy) which were all normal.

In the second group both FC and colonoscopy were requested on initial out-patient review. There were 23 patients in this group. 13 of 23 had normal FC and colonoscopy and no further investigations were done. 2 of 23 had abnormal FC (mean $271.5 \mu \mathrm{g} / \mathrm{gm}$ ) and colonoscopy. Both were diagnosed with IBD. 8 of 23 had raised FC (mean $171.25 \mu \mathrm{g}$ ) gm) but a normal colonoscopy. 5 of 8 had no further investigations done while 3 had small bowel investigations which were normal. 1 patient of these 3 was treated for presumed small bowel Crohn's due to raised FC despite normal capsule endoscopy with good effect.

In the third group colonoscopy was the initial investigation of choice and was found to be normal but FC was done later in view of persistent symptoms to look for small bowel inflammation. 18 patients fell in this group. 12 of 18 had normal FC and had no further investigations. 6 of 18 had raised FC (mean $114.33 \mu \mathrm{g} / \mathrm{gm}$ ). 3 patient's with raised FC had small bowel investigation done and all were normal.

Conclusion In conclusion FC was beneficial when negative. It provided reassurance to the clinicians and helped avoid invasive investigations. However when FC was positive clinical judgement and patient symptoms dictated the need for further investigations. None of the patients diagnosed with IBD had a negative FC.

Disclosure of Interest None Declared

\section{PTU-054 OUTCOMES FOLLOWING INVESTIGATION AND ELECTIVE WITHDRAWAL OF ANTI-TNF THERAPY IN CROHN'S DISEASE: A UK MULTICENTRE STUDY}

doi:10.1136/gutjnl-2013-304907.146

1."A J Brooks, ${ }^{2} \mathrm{~S}$ Sebastian, ${ }^{1} \mathrm{~K}$ Robinson, ${ }^{3} \mathrm{~L}$ Warren, ${ }^{1} \mathrm{~A}$ Wright, ${ }^{1} \mathrm{~A}$ M Marsh, ${ }^{2} \mathrm{H}$ Tsai, ${ }^{3} \mathrm{~F}$ Majeed, 'M E McAlindon, ${ }^{3 P} \mathrm{~J}$ Hamlin, 'A J Lobo. 'Sheffield Teaching Hospitals, Sheffield; ${ }^{2}$ Hull \& East Yorkshire NHS Trust, Hull, ${ }^{3}$ Leeds Teaching Hospitals NHS Trust, Leeds, UK

Introduction The impact of stopping anti-TNF for patients in clinical and/or endoscopic remission in routine clinical practise setting is uncertain. We aimed to evaluate clinical outcomes in patients who discontinued anti-TNF electively across 3 units in the Yorkshire \& Humber IBD Network, UK.

Methods Crohn's disease (CD) patients in whom anti-TNF (62 infliximab (IFX), 9 adalimumab (ADA)) was stopped electively following a planned assessment were included. All had been treated for $\geq 12$ months and followed-up for $\geq 3$ months following cessation of anti-TNF. Investigations at assessment prior to cessation included $\geq 1$ of; colonoscopy, colon capsule (CC), small bowel capsule (SBC), 
magnetic resonance enterography (MRE), barium study (BS), CRP and clinical assessment (CA).

Results Seventy-one patients (44 female; median age at diagnosis 24 years) were included with a median duration of IBD prior to antiTNF of 24 (0-264) months. Indications were severe active luminal (50/71), fistulating perianal $(18 / 71)$ and other fistulating disease $(3 / 71)$. The median treatment duration was 18 months (range $12-78)$ with 62 (87\%) on immunomodulators post anti-TNF withdrawal. Relapse rates within 90,180 and 365 days were $3 / 71(4.2 \%$ ), $14 / 67(21 \%)$ and $27 / 57(47 \%)$ respectively. In perianal disease alone, the relapse rate was $6 / 18(33 \%)$ at 1 year. 25 of those who relapsed were retreated with anti-TNF, with an overall recapture rate of $84 \%$. In those retreated with the same agent as previously withdrawn the response rate was $80 \%$. A further 5 were successfully retreated with ADA when IFX had been withdrawn. Those (6) who had a dose escalation in 6 months prior to withdrawal all relapsed.

Assessment practise changed following NICE guidance in 2010. Prior to this 5/15(33\%) stopping anti-TNF had a CA alone. Following NICE guidance $2 / 56$ (3.6\%) were assessed only by CA. Investigations to complement routine CA by Harvey Bradshaw Index (HBI), included $\geq 1$ of colonoscopy (52), CC (4), MRE (19), SBC (5), BS (2) and CRP (66). $\mathrm{HBI} \geq 4$ and a CRP of $\geq 5$ in the 6 months prior to formal assessment was observed in 26 patients. 14/26 (54\%) relapsed following cessation of anti-TNF (positive predictive value of $61 \%$ ). Further invasive investigations in this group were abnormal in 2 patients.

Conclusion In this UK cohort, elective withdrawal of anti-TNF was associated with a relapse rate of $48 \%$ after 12 months, with a high retreatment response rate. Due to NICE guidance, increased invasive assessment occurred, but the role of endoscopy and imaging to evaluate remission prior to withdrawal of anti-TNF needs further evaluation.

Disclosure of Interest A. Brooks: None Declared, S. Sebastian Conflict with: Dr Sebastian has participated in advisory boards and received speakers honoraria, educational and research grants from Abbott and MSD, K. Robinson: None Declared, L. Warren: None Declared, A. Wright: None Declared, A. Marsh: None Declared, H. Tsai Conflict with: Dr Tsai has participated in advisory boards and received speakers honoraria, educational and research grants from Abbott and MSD, F. Majeed: None Declared, M. McAlindon: None Declared, P. Hamlin Conflict with: Dr Hamlin has participated in advisory boards and received support for specialist nurses from Abbott and MSD, A. Lobo:None Declared

\section{PTU-055 LONG-TERM EFFICACY OF ADALIMUMAB FOR TREATMENT OF MODERATELY TO SEVERELY ACTIVE ULCERATIVE COLITIS}

doi:10.1136/gutjnl-2013-304907.147

1J-F Colombel, ${ }^{2} \mathrm{~W}$ J Sandborn, ${ }^{3} \mathrm{D}$ Wolf, ${ }^{4} \mathrm{R}$ Panaccione, ${ }^{5,} \mathrm{~A}$ Lazar, ${ }^{5} \mathrm{M}$ Kron, ${ }^{6} \mathrm{~A}$ M Robinson, ${ }^{6} \mathrm{R}$ Thakkar. 'Centre Hospitalier Universitaire de Lille, Lille, France; ${ }^{2}$ UCSD, La Jolla; ${ }^{3}$ Atlanta Gastro Assoc, Atlanta, United States; ${ }^{4}$ University of Calgary, Calgary, Canada; ${ }^{5} A b b V i e$ Deutschland, Ludwigshafen, Germany; ${ }^{6}$ AbbVie, North Chicago, United States
Introduction Objective To evaluate long-term efficacy of adalimumab (ADA) for patients with moderately to severely active ulcerative colitis (UC).

Methods The ADA UC development programme consists of two trials (ULTRA 1 and ULTRA 2) ${ }^{1,2}$ followed by an ongoing multicentre open-label $(\mathrm{OL})$ extension. Patients entering the extension on $\mathrm{OL}$ weekly ADA dosing continued on same. Patients entering the extension study from any blinded cohort (ADA or placebo [PBO]) or an OL cohort receiving ADA 40mg every other week (eow) received OL $\mathrm{ADA} 40 \mathrm{mg}$ eow. For patients entering from a blinded cohort, increase to $40 \mathrm{mg}$ weekly for flare or non-response was allowed at or after week 12. For patients entering from an OL cohort, increase to $40 \mathrm{mg}$ weekly for flare or non-response was allowed at or after week 12 for patients in clinical response at entry, or week 2 for patients with inadequate response at entry. Adjustments to concomitant medications including corticosteroids were allowed per protocol specifications. Partial Mayo score (PMS, Mayo score without endoscopy subscore) was collected at every study visit during the lead-in trials and the OL extension. Mean PMS over time through 3 years (172 weeks) from first dose of ADA was assessed using observed case method in the "any ADA" population (patients who received at least one dose of ADA in the lead-in or extension trials) using a data cut off of 16 December 2011. The proportion of patients in clinical remission per PMS (PMS $\leq 2$ with no subscore $>1$ ) at week 60 of the $\mathrm{OL}$ extension was assessed in the intent-to-treat (ITT) population (patients enrolled in the extension, excluding patients from sites non-compliant with good clinical practises), using non-responder imputation (NRI) to handle missing data.

Results The observed mean PMS at day of first dose of ADA was $5.9(\mathrm{~N}=992)$, and decreased over time through 172 weeks of treatment to $1.5(\mathrm{~N}=210$, Table). Of the 588 ITT patients from the leadin studies who enrolled into the OL extension, 325 (55.3\%, NRI) achieved clinical remission per PMS at week 60 of the OL extension. No new safety signals were observed.

Conclusion The results of the ongoing extension trial support clinically meaningful efficacy of adalimumab for the treatment of moderately to severely active UC, sustained for up to 3 years.

Disclosure of Interest J.-F. Colombel Shareholder of: Intestinal Biotech Development, Grant/Research Support from: AbbVie, Ferring, Merck \& Co, UCB Pharma, Giuliani SPA, Consultant for: AbbVie, Amgen, Biogen Idec Inc, Boehringer-Ingelheim, Inc., Bristol Meyers Squibb, Cellerix SL, Chemocentryx, Inc., Janssen, Cosmo Technologies, Ltd, Elan Pharmaceuticals, Inc., Roche, Giuliani SPA, Given Imaging, Glaxo Smith Kline, Immune Pharmaceuticals Ltd., ISR, Merck \& Co., Inc., Takeda, Neovacs SA, Ocerra Therapeutics, Inc., Pfizer Inc. Prometheus, Sanofi, Shire Pharmaceuticals, Synta Pharmaceutical Corporation, Takeda, Teva Pharmaceuticals, Therakos, TXcell, UCB Pharma, Speaker bureau with: AbbVie, Janssen, Falk Pharma, Ferring, Given Imaging, Merck \& Co., Inc., Shire Pharmaceuticals, UCB Pharma, W. Sandborn Grant/Research Support from: AbbVie, Bristol Meyers Squibb, Roche, Glaxo Smith Kline, Janssen, Takeda, Novartis, Pfizer, Procter and Gamble Pharmaceuticals, Shire

Abstract PTU-055 Table Mean PMS over time in the "any ADA" population, observed

\begin{tabular}{|l|c|c|c|c|c|c|c|c|c|c|c|c|}
\hline $\begin{array}{l}\text { Study } \\
\text { visit, } \\
\text { week }\end{array}$ & 0 & 4 & 8 & 16 & 32 & 52 & 64 & 88 & 112 & 136 & 160 & 172 \\
\hline $\mathrm{N}$ & 992 & 914 & 886 & 842 & 776 & 590 & 551 & 516 & 451 & 401 & 272 & 210 \\
\hline $\begin{array}{l}\text { PMS, } \\
\text { mean }\end{array}$ & 5.9 & 4.2 & 3.9 & 3.4 & 3.1 & 2.6 & 2.1 & 2.1 & 1.8 & 1.8 & 1.6 & 1.5 \\
\hline
\end{tabular}

